\title{
Improving the Quality of Clinical Pharmacy Services: A Process to Identify and Capture High-Value "Quality Actions"
}

\author{
Nicole Bruchet, Peter Loewen, and Jane de Lemos
}

\section{INTRODUCTION}

$\mathrm{P}$ atients are harmed and resources wasted because of underuse, overuse, and misuse of medications and treatments. ${ }^{1,2}$ To improve health services, governments and other key organizations use quality indicators. ${ }^{3,4}$ These indicators improve quality through 2 main mechanisms. First, the process of developing quality indicators allows standards, targets, and priorities to be set. Second, quality indicators are used to retrospectively measure and report various aspects of care, providing a framework that increases accountability, allows benchmarking, and identifies areas for improvement. ${ }^{5,6}$

Despite the widespread use of quality indicators to improve health services, the pharmacy profession has not widely adopted this concept for quality improvement in the clinical realm. We propose that this concept can be used to redesign the delivery of care. As pharmacists, we need to redefine what we need to be doing, find out whether we are doing it, and then use this information to find areas to improve.

Pharmacists cannot identify and manage all of the drugrelated problems that patients experience or are at risk of experiencing. Rather, the goal should be to maximize patient benefit with available resources. Pharmacists need to identify those drug-related problems for which management or prevention would result in the greatest benefit for as many patients as possible. In other words, they need to prioritize. In this article, we define a new concept that we call "quality actions" and describe a process to identify high-value quality actions for specific patient populations. Measurement consists of documenting whether or not a quality action has been considered or performed. This system will allow pharmacists to identify, measure, and report what they should be doing, which is fundamental to achieving improvement.

\section{BACKGROUND ON QUALITY INDICATORS AND QUALITY IMPROVEMENT}

Quality indicators are defined as measures based on standards of care that assess a particular health care process or outcome. ${ }^{6}$ They can be evidenced-based, or, if evidence is lacking, they can be determined by expert consensus. ${ }^{6}$ International and national agencies publish and use quality indicators to track performance and identify areas for improvement. ${ }^{3,4}$ For example, the Joint Commission has published 25 quality indicators for myocardial infarction, heart failure, pneumonia, and surgical care, ${ }^{3}$ and the US National Quality Forum has developed more than 200 quality indicators. ${ }^{7}$ If an intervention is associated with evidence of improved outcomes, setting a corresponding evidence-based standard (or process) that can be measured should lead to improvement in the process and its outcomes. ${ }^{89}$

The link between process and outcome is important. Many quality indicators focus on processes of care rather than on clinical outcomes because process indicators are usually more appropriate for generating improvements in quality. ${ }^{10}$ The reasons for this are critical but frequently misunderstood. Consider the following example. High-quality randomized controlled trials (RCTs) have shown that angiotensin-converting enzyme (ACE) inhibitor therapy reduces mortality among patients with systolic dysfunction. ${ }^{11}$ On this basis, a health care system might implement a process to ensure that "all patients with systolic dysfunction should receive ACE inhibitor therapy (assuming no contraindications)". The corresponding quality indicator would be "the proportion of patients with systolic dysfunction who have no contraindications to ACE inhibitors who actually receive an ACE inhibitor". This is a classic process measure. In this context, many clinicians and administrators 
want to measure mortality, since improvement in this outcome was the impetus for the quality program. Unfortunately, it is difficult to demonstrate improvement in outcomes, such as mortality, with corresponding improvements in processes. This is mainly because the studies that spurred the quality program were conducted under controlled circumstances designed to isolate the effect of the intervention being studied (in this case, ACE inhibitor therapy), and it is impossible to re-create those conditions in everyday practice. As a result, a multitude of confounders undermine efforts to show that using more ACE inhibitors in the health system population results in longer life for patients with systolic dysfunction. This does not mean that patients are not living longer-only that it is impossible to measure that effect in the absence of a concurrent control group, randomization, blinding, precise patient selection, and control over all the other changes occurring simultaneously in the system. Hence, it is appropriate to focus on doing the right things (those actions that have been shown by RCTs to be helpful), doing them more often, and measuring how often they are done.

Some pharmacists have begun to use quality indicators to measure the quality of drug therapy. ${ }^{12}$ For example, the "ideal medication intervention index" describes the proportion of eligible patients who receive proven interventions for their chronic medical conditions. Quality indicators have also been used to show that pharmacists' attendance at rounds is associated with increased use of evidenced-based interventions. ${ }^{13}$ However, instead of limiting quality indicators to the retrospective measurement of quality, we propose that they be integrated into routine care.

\section{QUALITY ACTIONS}

Measuring what pharmacists should be doing is fundamental to improving quality. ${ }^{14}$ Therefore, the actions needed to achieve a standard of care for specific conditions must be clearly defined. We call these "quality actions". Information should be captured as to whether these quality actions were considered and/or performed, as well as when and by whom they are supposed to be performed. This approach combines 2 important concepts. First, it incorporates the accepted elements of quality development: defining a measurable standard supported by evidence or expert opinion. Second, it supports the practical view that to improve care, the front-line providers should themselves define and capture whether the actions necessary to achieve the standard are considered and/or performed. ${ }^{15}$ Thus, the providers are prompted to consider these actions at the point of care and decision-making.

Quality health care is defined as "the degree to which health services for individuals and populations increase the likelihood of desired health outcomes and are consistent with professional knowledge". ${ }^{16}$ In addition, health care has 6 aims: to be safe, effective, patient-centred, timely, efficient, and equitable. ${ }^{17}$ These quality domains can be used as a conceptual framework to design a quality improvement model.

\section{DEVELOPING A PANEL OF HIGH-VALUE QUALITY ACTIONS}

By anticipating the medical conditions that are prevalent in specific populations, it is possible to develop panels of quality actions. The process can be applied to the patient population in any discipline (e.g., medicine, critical care, pediatrics, oncology, surgery). The 4 main criteria for quality are evidence base, effectiveness, safety, and efficiency (Table 1). The aggregate of these criteria can be considered a "quality aggregate". The criterion of efficiency has 2 subcriteria: modifiability and reliance on pharmacists to perform, which are important because pharmacists should align their efforts with what patients need them to do. "Modifiability" refers to processes or outcomes that pharmacists can reasonably be expected to alter. "Reliance on pharmacists to perform" refers to activities that the patient really needs the pharmacist to perform, as opposed to activities that could be done well by others on the team. Therefore, a total of 5 criteria form the quality aggregate. An action that meets all 5 criteria would have a higher value for conceptual quality aggregate than an action that meets only 3 of the criteria.

Three additional domains of quality do not contribute to the quality aggregate, but they are still important and may help in improving care: patient-centredness, timeliness, and equity (Box 1). Of these, the domain of patient-centredness is particularly important. Recently, Barry and others ${ }^{18}$ identified reasons why hospital pharmacists do not provide evidencebased care. One reason was a reluctance to address gaps in the care of patients with chronic diseases if the acute inpatient medical team preferred to defer management to the family doctor. To help close this gap, a quality action could be created that addresses patient-centredness through patient advocacy (Box 1). Furthermore, the actual performance of many quality actions should prompt the pharmacist to take a patient-centred approach by involving the patient in the process wherever feasible (Box 1).

\section{Value Index}

To prioritize their efforts, pharmacists need to consider the effort required to perform quality actions. We propose the following conceptual model:

$$
\text { Value index }=\frac{\begin{array}{c}
\text { Prevalence of problem in patient } \\
\text { population } \times \text { quality aggregate }
\end{array}}{\text { Effort required to manage }}
$$

where the "effort required to manage" can be manipulated through protocols, decision support systems, independent authority of pharmacists to order tests and modify therapy, etc. 
Table 1. Criteria for Selecting Quality Actions

\begin{tabular}{|c|c|c|}
\hline Criterion & Description & Recommendations \\
\hline Evidence base & $\begin{array}{l}\text { Considers the strength of evidence of drug } \\
\text { therapy interventions to improve outcomes } \\
\text { OR } \\
\text { Evidence exists that pharmacists are effective } \\
\text { in managing }\end{array}$ & $\begin{array}{l}\text { Quality actions should be supported by published } \\
\text { standards of care or by expert consensus, and the } \\
\text { corresponding level of evidence should be } \\
\text { characterizable. }\end{array}$ \\
\hline Effectiveness & $\begin{array}{l}\text { Categorized according to impact (e.g., reductions } \\
\text { in mortality, reductions in length of stay, and } \\
\text { down through the hierarchy of outcomes } \\
\text { relevant to a condition) }\end{array}$ & $\begin{array}{l}\text { A focus on effectiveness may be used to direct } \\
\text { pharmacists not only toward beneficial actions } \\
\text { but also away from actions or activities of less } \\
\text { effectiveness. * }\end{array}$ \\
\hline Safety & $\begin{array}{l}\text { Considers impact on safety by anticipating the } \\
\text { consequences of an adverse event on health } \\
\text { outcomes if event is not avoided }\end{array}$ & Actions that improve safety should be considered. \\
\hline Efficiency & $\begin{array}{l}\text { Avoidance of waste (e.g., money and other } \\
\text { resources) }\end{array}$ & $\begin{array}{l}\text { A focus on efficiency can include reducing costs } \\
\text { and ensuring that pharmacists spend time on } \\
\text { actions in proportion to the potential to modify } \\
\text { (or resolve) the problem and the degree of } \\
\text { reliance on the pharmacist to perform in the } \\
\text { context of other health care providers. }\end{array}$ \\
\hline Modifiability & $\begin{array}{l}\text { A link exists between a pharmacist performing } \\
\text { the action and a change in the health } \\
\text { outcome }\end{array}$ & $\begin{array}{l}\text { Example: Pharmacist ensures that any preadmission } \\
\text { medications taken for chronic conditions such as } \\
\text { congestive heart failure or chronic pulmonary } \\
\text { disease are continued and optimized. The patient's } \\
\text { condition is modifiable by the pharmacist's actions. } \\
\text { Example: Pharmacist spends time monitoring a } \\
\text { patient with influenza because of concern over } \\
\text { potential need to increase the antiviral dose. } \\
\text { However, there are no data to support the } \\
\text { assumption that increasing the dose of an } \\
\text { antiviral is associated with improvement in } \\
\text { outcome for a patient with influenza, so it may } \\
\text { be inefficient for a pharmacist to spend time } \\
\text { on this action. }\end{array}$ \\
\hline Reliance & $\begin{array}{l}\text { Actions for which the pharmacist is the most } \\
\text { suitable and/or most qualified practitioner }\end{array}$ & $\begin{array}{l}\text { Example: There may be little or no incremental } \\
\text { value in having a pharmacist with expertise in } \\
\text { postoperative pain management spend time on } \\
\text { this issue if other health care providers in the } \\
\text { unit also have this expertise. }\end{array}$ \\
\hline
\end{tabular}
${ }^{*}$ Avoidance of activities of limited effectiveness (e.g., routine measurement and evaluation of peak vancomycin levels in adults)
is also part of the definition of quality health care.

Once a candidate set of quality actions has been generated, each action can be evaluated according to its value index, and the set of actions prioritized accordingly. For example, an action involving a well-proven therapeutic intervention for a prevalent problem (e.g., statin therapy for patients with coronary artery disease) would have a high value index. However, if the target population was patients undergoing surgery and pharmacists had to rely on convincing reluctant surgeons to prescribe this type of therapy, the value index of this action would be considerably lower, because of the higher effort required to manage. On the other hand, if pharmacists had independent authority to initiate the therapy, the value index would increase substantially, because of a decrease in the denominator. Similarly, effort can be reduced by incorporating actions into protocols (e.g., risk assessment for preventing venous thromboembolism), which can free up time for pharmacists to have therapeutic discussions with patients and to spend more time on actions that are not easily incorporated into protocols.
By integrating a panel of high-value quality actions into daily care, pharmacists would provide evidenced-based, safe, effective drug therapy that is efficient and equitable for patients' needs.

\section{Other Uses and Benefits of a Panel of Quality Actions}

This model would allow new evidence to be immediately translated into practice. It would support professional development and accountability, and it might motivate pharmacists. Pharmacists working with their peers in similar practice settings could define the quality actions most relevant to their patients. Managers could recognize excellence and the value of pharmacists' care. They could also make more informed decisions about resource allocation because what the patient needs the pharmacist to do will have been defined. The manager would also be able to intuitively judge and compare relative importance and burden of complexity across different patient populations. 


\section{Box 1. Additional Domains of Quality to Be Reviewed}

\section{Patient-centredness}

- Reflects pharmacists' patient advocacy role (e.g., informing patients about gaps in care associated with proven interventions; asking patients if they want to know more about relative benefits and risks, rather than making assumptions about what they want or what physicians are willing to prescribe)

- Should be integrated in the performance of actions (e.g., involving patients in information-gathering and decisionmaking aspects of care)

- Example: Before recommending a change in a long-term medication, pharmacist asks patient to confirm how the drug is taken and whether the patient thinks the drug is working

\section{Timeliness}

- Addressed by use of authorities to permit more timely interventions to meet patient needs

- Example: Managers consider whether giving pharmacists independent authority to order tests or change doses would improve the timeliness of performing the quality action and take necessary steps to obtain institutional approval for pharmacists to perform this function

\section{Equity}

- Entails consideration of important actions with strong impacts in terms of their effectiveness, safety, or efficiency (cost-avoidance) for specific conditions in a specific population

- Reflects primordial definition of quality health care, which specifies the need to consider the population as well as the individual

- Ensures responsible allocation of limited pharmacist resources to issues where the largest benefit can be expected

- Allows provision of population-based pharmaceutical care and devotes resources in proportion to need (by anticipating the most important needs of the population)

\section{Comparison with Existing Systems for Documenting Clinical Actions}

Many pharmacy departments measure workload rather than quality. This work is often categorized and quantified according to the classification of drug-related problems (DRPs) proposed by Hepler and Strand. ${ }^{19,20}$ This framework may provide a structured approach to the delivery and teaching of pharmaceutical care ${ }^{19,20}$; however, it was not designed for assessing and prioritizing the quality of interventions or for facilitating quality improvement. It does not consider quality, as it provides no information on the extent to which clinical pharmacists are applying evidenced-based therapeutics. Even more problematic is the absence of the value of the intervention. For example, few would argue that the following pair of "add drug" resolutions for DRPs carry the same potential impact: adding antibiotic therapy for a patient with untreated bacteremia and adding a multivitamin preparation to a patient's drug regimen. We suggest that the quality actions model represents a philosophy of practice and a quality improvement system with significant advantages over existing systems. The quality actions model is derived from contemporary definitions of quality health care and its 6 aims. It requires consideration of the needs of the population (not just those of the individual), yet is explicitly patient-centred, since individuals are the focus of the actions. By operationalizing all the domains of quality, this model can help pharmacists and pharmacy departments to use their time and resources responsibly to maximize patient benefit and to deliver quality care, both to individual patients and to the overall population served.

\section{Potential Limitations of an Action-Oriented Approach to Quality Improvement}

The quality actions model has at least 3 potential limitations. First, quality actions target specific interventions for medical conditions or elements of practice. Consequently, less anticipated and as-yet-undefined high-value quality actions may be missed. However, the purpose is not to capture everything that clinical pharmacists do, but rather to focus on actions anticipated to be of highest value and quality in a specific practice area. Furthermore, the pharmacist can, through the rubric of the "value index", determine the appropriate amount of time to be spent on any issue in the context of all their patients' needs.

Second, focusing on defined quality actions could divert effort from other important but undefined activities. ${ }^{21}$ This unintended consequence may be minimized by identifying these activities, bringing them into the framework, and prioritizing them. This process may reveal activities on which pharmacists should be spending more or less time.

Third, quality actions are not "denominator-oriented" and so do not support measurement of the proportion of times an intervention is appropriately performed in an eligible population. Therefore, if resources permit and where the eligible patient population can be retrospectively identified, the quality action can be converted to a quality indicator using methods already reported. ${ }^{12}$ This will be useful if underperformance of high-value quality actions is suspected. For example, Gorman and others ${ }^{22}$ identified underuse of calculation of a clinical pulmonary infection score and failure to stop antibiotics at 3 or 8 days as an opportunity for improvement for patients with ventilator-associated pneumonia. In particular, antibiotics were stopped at 8 days for only $12 \%$ of patients. ${ }^{22}$ Therefore, a quality action for ventilator-associated pneumonia would be calculation of the clinical pulmonary infection score with assessment at 3 and 8 days and cessation of therapy if appropriate.

Selective review of other quality indicators may justify additional resources. In particular, underuse of quality actions may be due to inadequate pharmacist resources. However, unlike the pharmacy distribution system, in which delays in 
medication delivery or errors in medication dispensing are readily apparent, missed opportunities to perform quality actions are not visible for clinical services. Increasing the visibility of these missed opportunities may help to justify additional resources.

Implementation of this model may be challenging. Pharmacists may be reluctant to record when an action was taken because of discomfort with a perceived "checklist" approach. However, checklists have been shown to improve care in a variety of settings. ${ }^{23,24}$ Pharmacists and managers may have discomfort with the associated increase in accountability. Effort will be required to develop the necessary infrastructure, but the leaders of our profession should understand that we need to meet our obligation to continually improve quality, ensure effective use of resources, and support pharmacists in delivering better care.

Finally, some pharmacists may feel that implementing the model would require precise quantification of the quality aggregate and the value index. However, such a scoring system is not a prerequisite for adopting the model. The quality actions and the value index form a conceptual framework that is actionable now by any pharmacist, in any practice area, to improve the care delivered. In its current form, this framework should help pharmacists to start thinking objectively in terms of quality actions and to start using the value index conceptually, to prioritize unanticipated issues and initiatives. Although they incorporate a formula, these concepts should not require precise mathematical expression to operationalize them in every situation. The framework should also allow most pharmacist teams to develop their own population-specific panels of quality actions. Thoughtful discussion of the reasons for disagreement about the quality aggregate or the effort required to manage will be needed to reveal opportunities for improvement (Box 2). This is a critical step in the process, which cannot be replaced by a scoring system. Nonetheless, a scoring system could improve the transparency of the ranking and decisionmaking process for selecting quality actions, as it would ensure that the domains of the quality aggregate and the components of the value index have been properly considered. It would more readily reveal where disagreement lies. We believe that a scoring system, based on levels of evidence, efficacy, and safety (as shown in Table 1), could be developed for the quality aggregate and other model components to produce the value index. We hope that introducing these concepts here will foster dialogue to inform such research.

\section{CONCLUSIONS}

Using a quality improvement model based on "quality actions" may represent a significant improvement over DRPand workload-based methods to guide and improve the delivery of care by pharmacists. Pharmacist teams can use the

\section{Box 2. Potential Reasons for Differences in Choice of Quality Actions*}

Lack of knowledge or uncertainty of interpretation

- Pharmacists may be unaware of recent literature or may not have had time to interpret it properly

- Pharmacists may be aware of literature but may not fully understand expected effect on the patient

- Discussion and review of literature as a group may help in standardizing interpretation

\section{Lack of confidence}

- Pharmacists may be aware but may lack confidence

- Pharmacists may be unaware of what colleagues are doing but could be influenced by peers

- Opportunity to shine light on best practice

- Allows pharmacists who are comfortable in the area to support others with less confidence

- Managers could consider educational program or mentorship to improve confidence and comfort level

\section{Conditioning on the part of the prescriber (inertia)}

- Pharmacists may have stopped making an appropriate recommendation because the prescriber routinely does not accept the recommendation (e.g., a surgeon might insist on providing 3 days of postoperative antibiotic prophylaxis although this is not indicated)

- Managers can support pharmacists by offering to engage in discussion with prescribers and any other institutional groups with a mandate for action in the area of concern (e.g., antimicrobial stewardship)

Lack of communication skills to convey rationale to prescribers

- Managers can consider how to help the pharmacist build better skills to facilitate changes in prescribers' behaviour

*Identified through discussion with teams of pharmacists practising in the same area. Note that reasons behind variance should be explored, through discussion of the quality action panel content (and associated domains of quality aggregate, expression of effort required to manage and known prevalence of condition).

value index to design and prioritize their own panels of quality actions. Further work is needed to collaboratively develop population-specific panels and efficient documentation and reporting processes and to determine whether pharmacists and managers consider this an improved process.

\section{References}

1. Chassin MR, Galvin RW. The urgent need to improve health care quality: Institute of Medicine National Roundtable on Health Care Quality. JAMA 1998;280(11):1000-1005.

2. Hayward RA, Asch SM, Hogan MH, Hofer TP, Kerr EA. Sins of omission: getting too little medical care may be the greatest threat to patient safety. $J$ Gen Intern Med 2005;20(8):686-691.

3. Improving America's hospitals: The Joint Commission's annual report on quality and safety 2008. Oakbrook Terrace (IL): The Joint Commission; 2008 [cited 2009 Jul 15]. Available from: www.jointcommissionreport.org

4. Measuring for quality improvement. London (UK): UK Department of Health; 2008 [cited 2009 Jul 15]. Available from: www.ic.nhs.uk/ services/measuring-for-quality-improvement

5. Setting key targets for executive agencies: a guide. London (UK): HM Treasury; 2003 [cited 2009 Jul 15]. Available from: www.ccas.min-financas.pt/ documentacao/setting-key-targets-for-executive-agencies-a-guide 
6. Mainz J. Defining and classifying clinical indicators for quality improvement. Int J Qual Health Care 2003;15(6):523-530.

7. NQF-endorsed ${ }^{\circledR}$ standards. Washington (DC): National Quality Forum; [cited 2009 Jul 15]. Available from: www.qualityforum.org/Measures_ List.aspx

8. Jha AK, Perlin JB, Kizer KW, Dudley RA. Effect of the transformation of the Veterans Affairs Health Care System on the quality of care. $N$ Engl J Med 2003;348(22):2218-2227.

9. Hermanides HS, Hulscher MEJL, Schouten JA, Prins JM, Geerlings SE. Development of quality indicators for the antibiotic treatment of complicated urinary tract infections: a first step to measure and improve care. Clin Infect Dis 2008;46(5):703-711.

10. Rubin HR, Pronovost P, Diette GB. The advantages and disadvantages of process-based measures of health care quality. Int $J$ Qual Health Care 2001;13(6):469-474

11. Cohn JN, Johnson G, Ziesche S, Cobb F, Francis G, Tristani F, et al. A comparison of enalapril with hydralazine-isosorbide dinitrate in the treatment of chronic congestive heart failure. N Engl J Med 1991;325(5):303-310.

12. Huang C, Loewen P, Pelletier T, Slater J, Chung M. Implementation of proven interventions in general medical inpatients: development and evaluation of a new quality indicator for drug therapy. Qual Saf Health Care 2008;17(4):269-274.

13. Makowsky MJ, Koshman SL, Midodzi WK, Tsuyuki RT. Capturing outcomes of clinical activities performed by a rounding pharmacist practicing in a team environment: The COLLABORATE Study. Med Care 2009;47(6):642-650.

14. Brook RH, McGlynn EA, Cleary PD. Quality of health care. Part 2: Measuring quality of care. N Engl J Med 1996;335(13):966-970.

15. Werner RM, McNutt R. A new strategy to improve quality: rewarding actions rather than measures. JAMA 2009;301(13):1375-1377.

16. Schoenbaum S, Sundwall DN, Bergman D, Buckle JM, Chernov A, George J, et al. Using clinical practice guidelines to evaluate quality of care. AHCPR Publ No. 95-0046. Rockville (MD): US Department of Health and Human Services, Public Health Service and Agency for Health Care Policy and Research; 1995 Mar.

17. Institute of Medicine, Committee on Quality of Health Care in America. Crossing the quality chasm. A new health system for the 21st century. Washington (DC): National Academy Press; 2005.

18. Barry A, Loewen P, de Lemos J, Lee KG. Reasons for non-use of proven interventions for hospital inpatients: pharmacists' perspectives. Can J Hosp Pharm 2009;62(5):381-385.
19. Strand LM, Morley PC, Cipolle RJ, Ramsey R, Lamsam GD. Drug-related problems: their structure and function. DICP 1990;24(11):1093-1097.

20. Hepler CD, Strand LM. Opportunities and responsibilities in pharmaceutical care. Am J Hosp Pharm 1990;47(3):533-543.

21. Goddard M, Davies HTO, Dawson D, Mannion R, McInnes F. Clinical performance measurement: part 2 - avoiding the pitfalls. $J R$ Soc Med 2002;95(11):549-551.

22. Gorman SK, Stewart LMM, Slavik RS, de Lemos J, Chittock D, Dhingra VK, et al. Identifying missed opportunities to curtail antimicrobial therapy for presumed ventilator-associated pneumonia using the clinical pulmonary infection score. Can J Hosp Pharm 2009;62(3):217-225.

23. Pronovost P, Needham D, Berenholtz S, Sinopoli D, Chu H, Cosgrove S, et al. An intervention to decrease catheter-related bloodstream infections in the ICU. N Engl J Med 2006;355(26):2725-2732.

24. Haynes AB, Weiser TG, Berry WR, Lipsitz SR, Breizat AH, Dellinger P, et al. A surgical safety checklist to reduce mortality and morbidity in a global population. N Engl J Med 2009;360(5):491-499.

Nicole Bruchet, BSc, BSc(Pharm), PharmD, is with Pharmacy Services, Kelowna General Hospital, Kelowna, British Columbia. At the time of writing, she was a Doctor of Pharmacy student in the Faculty of Pharmaceutical Sciences, University of British Columbia, Vancouver, British Columbia.

Peter Loewen, BSc(Pharm) PharmD, is with Pharmacy Services, Vancouver Coastal Health-Providence Health Care, part of Lower Mainland Pharmacy Services, and the Faculty of Pharmaceutical Sciences, University of British Columbia, Vancouver, British Columbia.

Jane de Lemos, BPharm, PharmD, MSc, is with Pharmacy Services, Vancouver Coastal Health-Providence Health Care, part of Lower Mainland Pharmacy Services, Vancouver, British Columbia.

\section{Address correspondence to:}

Dr Jane de Lemos

Pharmacy Services

Vancouver Coastal Health-Providence Health Care

Third Floor, 865 West 10th Avenue

Vancouver BC V5Z $1 \mathrm{M} 9$

e-mail: jane.delemos@vch.ca 